\title{
Brain Rehabilitation Assessment and InterventioN (BRAIN): Delivering Efficacious Training at Home
}

\author{
Harry Hallock \\ Department of Neurology, \\ Charité - Universitätsmedizin \\ Berlin; Berlin School of Mind \\ and Brain, Humboldt-Universität \\ zu Berlin. \\ Berlin, Germany \\ hariharan- \\ michael.hallock@charite.de
}

\author{
Amit Lampit \\ Department of Neurology, \\ Charité - Universitätsmedizin \\ Berlin; Berlin School of Mind \\ and Brain, Humboldt-Universität \\ zu Berlin; Academic Unit for \\ Psychiatry of Old Age, University \\ of Melbourne. \\ Berlin, Germany \\ amit.lampit@charite.de
}

\author{
Joseph Kuchling \\ Department of Neurology, \\ Charité - Universitätsmedizin \\ Berlin; Berlin School of Mind \\ and Brain, Humboldt-Universität \\ zu Berlin, \\ Berlin, Germany \\ joseph.kuchling@charite.de
}

\author{
Carsten Finke \\ Department of Neurology, \\ Charité - Universitätsmedizin \\ Berlin; Berlin School of Mind \\ and Brain, Humboldt-Universität \\ zu Berlin, \\ Berlin, Germany \\ carsten.finke@charite.de
}

\begin{abstract}
Computerised cognitive training is an efficacious strategy for cognitive impairment across the lifespan and neurodegenerative disorders, a pressing and unmet public health challenge. Yet efficacy is strongly related to key intervention design factors, and we currently do not have the tools to deliver clinical-grade cognitive training at scale. BRAIN, a digital diagnostic and rehabilitation tool, aims to close this implementation gap by facilitating remote clinician-led assessment, training monitoring of cognitive performance using novel personalization and communication solutions.
\end{abstract}

Keywords - cognitive assessment, cognitive training, interventions design.

\section{INTRODUCTION}

Cognitive impairment is common in ageing and across psychiatric and neurological disorders. Insidious cognitive impairment will typically lead to dementia, a leading cause of disability worldwide and the leading cause of death in the UK [1]. As such, developing interventions to reduce the burden of cognitive impairment and delay cognitive decline are a global health priority.

In neurological diseases where cognitive impairment is present and impacts on quality of life, such as multiple sclerosis, cognitively stimulating activities have been shown to improve cognition [2]. Furthermore, given that cognitive inactivity is a major modifiable risk factor for dementia [3], and engagement in complex mental activity has been associated with a decreased risk of incident dementia [4], an intervention which specifically trains cognition may be warranted.

\section{Computerised Cognitive Training}

Computerised cognitive training (CCT) is a common intervention used in trials targeting prevention and rehabilitation of cognitive decline in a variety of different populations. Specifically, a strong body of evidence suggests that CCT is efficacious for cognition in non-demented older adults $[5,6]$. There is also sufficient evidence illustrating the efficacy of CCT for improving cognition in mild cognitive impairment [6], Parkinson's disease [7] and major depression [8].

This research is funded by the German Federal Ministry of Education and Research (BMBF)
CCT involves repeated practice on inherent problems using standardized tasks that target specific cognitive processes, using interactive and adaptive exercises [9]. These advantages suggest that CCT might be a more scalable intervention compared to more traditional approaches. However, the field has yet to see large-scale, long-term clinical trials or implementation studies. This is likely due to the limited efficacy and high attrition rates commonly seen in trials of home-based CCT. These are likely to be a result of low patient motivation, limited clinician supervision and communication, poor outcome variables, an inflexible intervention and inadequate integration within the platform.

\section{A Digital Solution}

To overcome the current limitations of home-based CCT, a digital diagnostic and rehabilitation tool, Brain Rehabilitation Assessment and InterventioN (BRAIN) is developed. The platform, which will be used by both patients and clinicians, aims to integrate neuropsychological assessments, CCT, monitoring, remote supervision and motivational support for home-based delivery. As such it aims to close the current implementation gap in the field, whilst also improving the accessibility and regulation of CCT as a therapy.

\section{INTEGRATION}

Patients are enrolled into the platform by their clinician and administered baseline neuropsychological assessments. The clinician decides which assessments will be administered or selects a pre-defined assessment battery based on their patient's clinical presentation (e.g. amnestic-MCI or concussion assessment). Following baseline assessment, the clinician then has the option (i) to administer a CCT plan (Fig. 1) based on the patient's assessment performance, (ii) to select pre-defined training plans designed for different diseases (e.g. amnesticMCI plan, concussion plan, or Parkinson's plan) or (iii) to select pre-defined training plans designed to focus on specific domains (e.g. working memory plan, speed plan, attention plan). In addition, all default settings for training plans can be modified by the clinician. Performance on the training plans is then used to tailor and update the plan at pre-specified intervals. Unless overridden by the clinician, the system will provide a set level of motivation and social support cues to the patient based on their performance, their diagnosis and changes in their 
cognitive profile with time. These cues include motivational messages within the exercises or notifications in their dashboard.

One of the most difficult tasks for clinicians administering remote therapy is effective time-management, i.e., meeting the different supervision needs of multiple patients, and knowing if and when to intervene. BRAIN will provide real-time supervision and monitoring of patients based on a pre-defined logic and can create automated alerts that are sent to the clinician. For example, the clinician can choose to be notified when a patient does not complete a scheduled training session or when training performance begins to plateau or decline. In addition, the system will provide automated alerts to the patient. For example, the system will ask the patient if they need assistance in case of an unexpected drop in performance on a particular exercise. Based on the patient's response, the system either provides a pre-designed solution (e.g. a tutorial or further instructions) or notifies the clinician. Furthermore, if patients have difficulties or questions throughout the intervention, they can lodge a support request in the system. This is automatically prioritized based on the clinician's preferences, that can be individually defined prior to and during training period, thus allowing the clinician to attend to urgent requests first.

\section{CONCLUSION}

This platform aims to cognitively rehabilitate patients by simultaneously providing patients with support and guidance, and clinicians with the tools and flexibility needed to provide meaningful cognitive interventions to numerous patients with diverse needs.

\section{ACKNOWLEDGMENT}

The authors thank their collaborating partner NeuroNation ${ }^{\circledR}$, for their software expertise in developing BRAIN.

\section{REFERENCES}

Office for National Statistics. (30 January 2019). Deaths registered in England and Wales (Series DR): 2017. Available: https://www.ons.gov.uk/peoplepopulationandcommunity/birthsdea thsandmarriages/deaths/bulletins/deathsregisteredinenglandandwal esseriesdr/2017

[2] E. Dardiotis et al., "Efficacy of computer-based cognitive training in neuropsychological performance of patients with multiple sclerosis: A systematic review and meta-analysis," (in eng), Mult Scler Relat Disord, vol. 20, pp. 58-66, Feb 2018.

[3] D. E. Barnes and K. Yaffe, "The projected effect of risk factor reduction on Alzheimer's disease prevalence," (in eng), Lancet Neurol, vol. 10, no. 9, pp. 819-28, Sep 2011.

[4] M. J. Valenzuela and P. Sachdev, "Brain reserve and dementia: a systematic review," (in eng), Psychol Med, vol. 36, no. 4, pp. 44154, Apr 2006.

[5] A. Lampit, H. Hallock, and M. Valenzuela, "Computerized cognitive training in cognitively healthy older adults: a systematic review and meta-analysis of effect modifiers," (in eng), PLoS Med, vol. 11, no. 11, p. e1001756, Nov 2014.

[6] Nicole T.M. Hill, Loren Mowszowski, Sharon L. Naismith, Verity L. Chadwick, Michael Valenzuela, and Amit Lampit, "Computerized Cognitive Training in Older Adults With Mild Cognitive Impairment or Dementia: A Systematic Review and Meta-Analysis," American Journal of Psychiatry, vol. 174, no. 4, pp. 329-340, 2017.

[7] I. H. K. Leung, C. C. Walton, H. Hallock, S. J. G. Lewis, M. Valenzuela, and A. Lampit, "Cognitive training in Parkinson disease: A systematic review and meta-analysis," (in eng), Neurology, vol. 85, no. 21, pp. 1843-51, Nov 242015.

[8] J. N. Motter, M. A. Pimontel, D. Rindskopf, D. P. Devanand, P. M. Doraiswamy, and J. R. Sneed, "Computerized cognitive training and functional recovery in major depressive disorder: A meta-analysis," (in eng), J Affect Disord, vol. 189, pp. 184-91, Jan 12016.

[9] N. Gates and M. Valenzuela, "Cognitive exercise and its role in cognitive function in older adults," (in eng), Curr Psychiatry Rep, vol. 12, no. 1, pp. 20-7, Feb 2010.

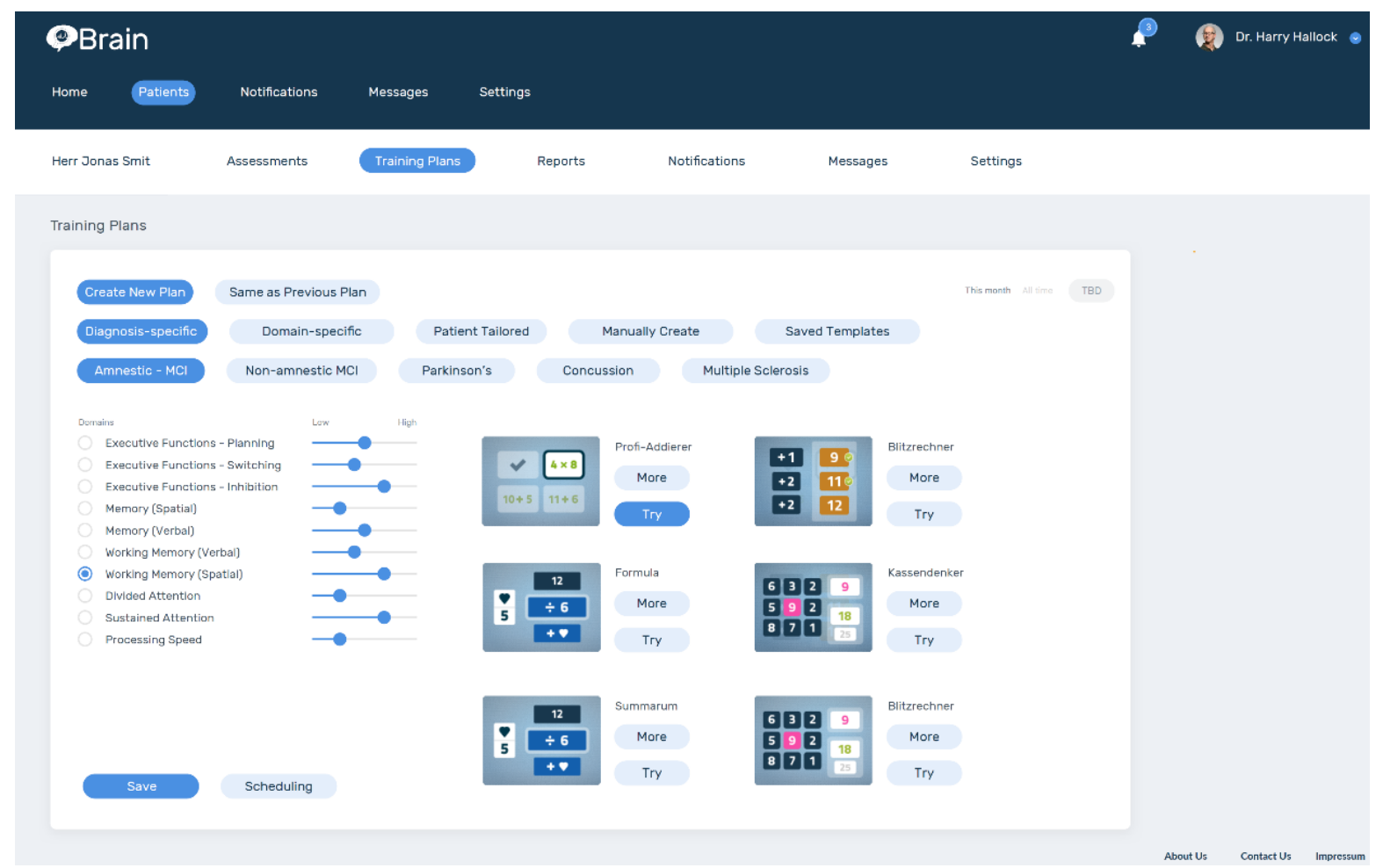

Fig 1. Prototype of the Training Plans page in the clinician interface view in BRAIN 